\title{
Association Between Convenience of Transportation and Unmet Healthcare Needs of Rural Elderly in Korea
}

\author{
Youngeun Choi' ${ }^{1}$ Kiryong Nam ${ }^{1}$, Chang-yup Kim ${ }^{2,3}$ \\ ${ }^{1}$ Department of Preventive Medicine, Graduate School of Public Health, Seoul National University, Seoul, Korea; ${ }^{2}$ Department of Public Health \\ Science, Graduate School of Public Health, Seoul National University, Seoul, Korea; Institute of Health and Environment, Seoul National University, \\ Seoul, Korea
}

Objectives: In rural areas of Korea, where public transportation infrastructure is lacking and alternative systems are poor, the elderly experience inconveniences in using healthcare, although their need is high. This study aimed to analyze the association between the convenience of transportation and unmet healthcare needs among the rural elderly.

Methods: The data used were collected in the 2016 Community Health Survey among rural elderly individuals aged 65 or older. Dependent variable was the unmet healthcare needs, explanatory variable was the convenience of transportation. The elderly were divided into 3 groups: with no driver in the household, with a driver, and the elderly individual was the driver (the self-driving group). Covariates were classified into predisposing, enabling, and need factors. They included gender, age, education, income, economic activity, household type, motor ability, subjective health level, number of chronic diseases, anxiety/depression, and pain/discomfort. The data were analyzed using logistic regression and stratification.

Results: A significant association was found between the convenience of transportation and unmet healthcare needs. When examined unadjusted odds ratio of the group with a driver in the household, using the group with no driver as a reference, was 0.61 (95\% confidence interval $[\mathrm{Cl}], 0.54$ to 0.68 ), while that of the self-driving group was 0.34 ( $95 \% \mathrm{Cl}, 0.30$ to 0.38 ). The odds ratios adjusted for all factors were $0.69(95 \% \mathrm{Cl}, 0.59$ to 0.80$)$ and $0.79(95 \% \mathrm{Cl}, 0.67$ to 0.91$)$.

Conclusions: We confirmed a significant association between inconvenient transportation and unmet healthcare needs among the rural elderly even after adjustment for existing known factors. This implies that policies aimed at improving healthcare accessibility must consider the means of transportation available.

Key words: Unmet healthcare needs, Rural elderly, Health services accessibility, Transportation, Healthcare disparity

Received: June 28, 2019 Accepted: October 1, 2019

Corresponding author: Chang-yup Kim, MD, PhD Department of Public Health Science, Graduate School of Public Health, Seoul National University, 1 Gwanak-ro, Gwanak-gu, Seoul 08826, Korea

E-mail: cykim@snu.ac.kr

This is an Open Access article distributed under the terms of the Creative Commons Attribution Non-Commercial License (http://creativecommons.org/licenses/by$\mathrm{nc} / 4.0 / /$ which permits unrestricted non-commercial use, distribution, and reproduction in any medium, provided the original work is properly cited.

\section{INTRODUCTION}

Healthcare accessibility refers to equality in the use of healthcare regardless of other factors that do not relate to healthcare needs (such as payment ability, residence, and age) when equal healthcare needs exist [1]. Health inequality makes groups with less accessibility more disadvantaged in terms of health and reduces opportunities to be healthy [2]. Barriers to healthcare accessibility appear in various ways depending on the situation faced; similarly, unmet healthcare 
needs can also manifest in different ways. For instance, temporary workers have a high level of unmet healthcare needs [3] as they have spent little time in Korean society, migrant women experience difficulty due to language barriers [4], and people with disabilities encounter various types of barriers depending on the type of disability [5]. The barriers for the rural elderly are associated with several problems. Although healthcare needs increase with age, the rural elderly experience difficulties due to factors such as a lack of regional medical resources, an unbalanced distribution of those resources, poor infrastructure, and the vulnerable condition due to ageing $[6,7]$.

Most policies related to healthcare accessibility have focused on lowering barriers from the supply side [8], and Korean policies have been no exception. However, it is logical to lower the barriers on the demand side [9], as well as on the supply side, to better meet the healthcare needs of the demanders (patients). The physical barriers to healthcare accessibility from the supply side involve allocation of medical facilities and resources, whereas from the demand side, barriers include the transportation process from households to healthcare institutions. Unless the means of transportation from residences to healthcare institutions are improved, even if the allocation of medical resources (such as medical facilities or personnel) is increased, elderly individuals with poor motor ability will still face a barrier [10] that may be too high to surmount despite a short distance or a short calculated travel time.

In other countries, studies have confirmed that transportation was the most influential determinant of healthcare accessibility $[11,12]$. Those studies identified an association between means of transportation and healthcare use, as can be exemplified by the association between driving as an elderly individual and the regular check-up and management of chronic conditions [10]. However, few Korean studies [13] have expressed interest in means of transportation for healthcare use for the elderly, although studies have investigated the inconvenience of transportation of rural area $[14,15]$ and travel time and distance to healthcare institutions [16-18]. Although some qualitative studies have found that a lack of transportation limits healthcare use by the elderly $[2,19]$, no research has yet explored the association between means of transportation and the healthcare use or unmet healthcare needs of the rural elderly.

The first step in responding to health inequalities is measuring them [20]. To prepare measures and policies aimed at low- ering the barriers to healthcare accessibility for the rural elderly in Korea, it is necessary to first assess the presence and extent of any differences in the unmet healthcare needs of that population based on means of transportation apart from the factors that have been identified so far. This study aimed to analyze the association between the convenience of transportation and the unmet healthcare needs of the rural elderly with the goal of making an evidence for suggestions to improve healthcare accessibility and to address problems relating to unequal access.

\section{METHODS}

\section{Data Source}

This study used 2016 data from the Community Health Survey, which was conducted by the Korea Centers for Disease Control and Prevention. From the raw data, which included 228452 adults aged 19 or older from across the nation, only the data from health centers in gun (county) areas was extracted (with the health centers in the guns of Busan, Daegu, Incheon, and Ulsan excluded from the analysis). In our study, data from 28191 elderly individuals aged 65 or older among 68150 adults from 77 health centers were analyzed.

\section{Dependent Variable}

The dependent variable in this study was the level of unmet healthcare needs. Individuals who replied "yes" to the question "Over the past year, have you ever not been able to visit the hospital or clinic (not including a dental clinic) even though you wanted to visit?" were said to have unmet healthcare needs, while those who replied "no" were said to have no unmet healthcare needs. The variables used to describe the reasons for unmet healthcare needs were set by the reply to the question "If you have had such an experience, what reason prevented you from visiting the hospital(s) or clinic(s) you wanted to visit?"

\section{Explanatory Variable}

For the variable describing convenience of transportation, a questionnaire was used to ascertain whether driving was available to the elderly individual. The participants were divided into 3 groups (a self-driving group, a group with a driver who was not the elderly, and a group with no driver in the household) for analysis. 


\section{Covariates}

Existing known factors were categorized according to the concept of the predisposing, enabling, and need factors from Andersen's healthcare utilization model [21]. The predisposing factors included gender, age, and education level, while the enabling factors included household income, economic activity, household type, and motor ability. Finally, the need factors included subjective health level, the number of chronic dis- eases of the individual, anxiety or depression, and pain or discomfort.

\section{Statistical Analysis}

A frequency analysis and the chi-square test were conducted to investigate general characteristics of the research subjects (the rural elderly) as well as their status regarding unmet healthcare needs.

Table 1. Characteristics of the rural elderly and distribution of unmet healthcare needs

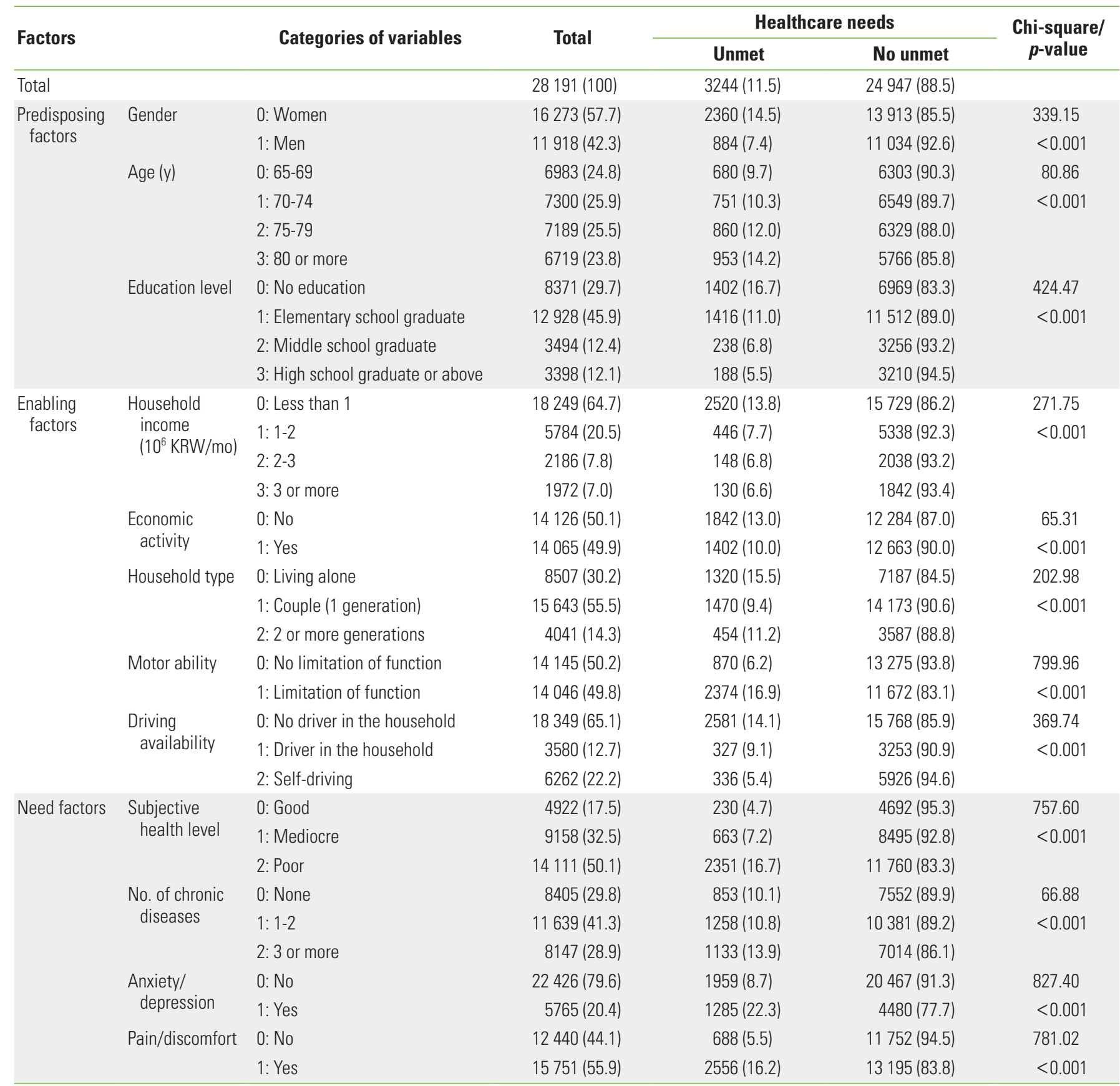

Values are presented as number (\%). 
Logistic regression analysis was conducted to investigate the association between the convenience of transportation and the unmet healthcare needs. Spearman correlation analysis was used to check multicolinearity. By conducting logis- tic regression analysis for the predisposing, enabling, and need factors of Andersen's healthcare utilization model, the odds ratio (OR) before and after adjustment were calculated.

Table 2. Results of regression analysis on factors influencing unmet healthcare needs

\begin{tabular}{|c|c|c|c|c|c|}
\hline \multirow{2}{*}{ Factors } & \multirow{2}{*}{ Explanation of variables } & \multirow{2}{*}{ Total (n) } & \multirow{2}{*}{$\begin{array}{c}\text { Unmet healthcare } \\
\text { needs (n) }\end{array}$} & \multicolumn{2}{|c|}{ Adjustment } \\
\hline & & & & Before & After \\
\hline Total & & 28191 & 3244 & & \\
\hline \multicolumn{6}{|l|}{ Predisposing Factors } \\
\hline \multirow[t]{2}{*}{ Gender } & 0: Women & 16273 & 2360 & 1.00 (reference) & 1.00 (reference) \\
\hline & 1: Men & 11918 & 884 & $0.47(0.44,0.51)^{* * *}$ & $0.79(0.71,0.88)^{* * *}$ \\
\hline \multirow[t]{4}{*}{ Age (y) } & $0: 65-69$ & 6983 & 680 & 1.00 (reference) & 1.00 (reference) \\
\hline & 1: $70-74$ & 7300 & 751 & $1.06(0.95,1.18)^{* * *}$ & $0.76(0.67,0.85)^{* * *}$ \\
\hline & $2: 75-79$ & 7189 & 860 & $1.26(1.13,1.40)^{* * *}$ & $0.71(0.64,0.81)^{* * *}$ \\
\hline & 3: 80 or more & 6719 & 953 & $1.53(1.38,1.70)^{* * *}$ & $0.72(0.64,0.83)^{* * *}$ \\
\hline \multirow[t]{4}{*}{ Education level } & $0:$ No education & 8371 & 1402 & 1.00 (reference) & 1.00 (reference) \\
\hline & 1: Elementary school graduate & 12928 & 1416 & $0.61(0.56,0.66)^{* * *}$ & $0.79(0.72,0.86)^{* * *}$ \\
\hline & 2: Middle school graduate & 3494 & 238 & $0.36(0.31,0.42)^{* * *}$ & $0.67(0.57,0.79)^{* * *}$ \\
\hline & 3: High school graduate or above & 3398 & 188 & $0.29(0.24,0.34)^{* * *}$ & $0.73(0.60,0.88)^{* * *}$ \\
\hline \multicolumn{6}{|l|}{ Enabling factors } \\
\hline \multirow{4}{*}{$\begin{array}{l}\text { Household income } \\
\left(10^{6} \mathrm{KRW} / \mathrm{mo}\right)\end{array}$} & 0 : Less than 1 & 18249 & 2520 & 1.00 (reference) & 1.00 (reference) \\
\hline & $1: 1-2$ & 5784 & 446 & $0.52(0.47,0.58)^{* * *}$ & $0.76(0.68,0.86)^{* * *}$ \\
\hline & $2: 2-3$ & 2186 & 148 & $0.45(0.38,0.54)^{* * *}$ & $0.71(0.59,0.86)^{* * *}$ \\
\hline & $3: 3$ or more & 1972 & 130 & $0.44(0.36,0.53)^{* * *}$ & $0.68(0.55,0.83)^{* * *}$ \\
\hline \multirow[t]{2}{*}{ Economic activity } & $0:$ No & 14126 & 1842 & 1.00 (reference) & 1.00 (reference) \\
\hline & 1: Yes & 14065 & 1402 & $0.74(0.68,0.79)^{* * *}$ & $1.23(1.13,1.34)^{* * *}$ \\
\hline \multirow[t]{3}{*}{ Household type } & 0: Living alone & 8507 & 1320 & 1.00 (reference) & 1.00 (reference) \\
\hline & 1: Couple (1 generation) & 15643 & 1470 & $0.56(0.52,0.61)^{* * *}$ & $0.90(0.82,0.99)^{*}$ \\
\hline & 2: 2 or more generations & 4041 & 454 & $0.69(0.61,0.77)^{* * *}$ & $1.19(1.03,1,37)^{*}$ \\
\hline \multirow[t]{2}{*}{ Motor ability } & 0 : No limitation of function & 14145 & 870 & 1.00 (reference) & 1.00 (reference) \\
\hline & 1: Limitation of function & 14046 & 2374 & $3.10(2.85,3.36)^{* * *}$ & $1.50(1.35,1.66)^{* * *}$ \\
\hline \multirow[t]{3}{*}{ Driving availability } & 1: No driver in household & 18349 & 2581 & 1.00 (reference) & 1.00 (reference) \\
\hline & 2: Driver in household & 3580 & 327 & $0.61(0.54,0.68)^{* * *}$ & $0.69(0.59,0.80)^{* * *}$ \\
\hline & 3: Self-driving & 6262 & 336 & $0.34(0.30,0.38)^{* * *}$ & $0.79(0.67,0.91)^{* *}$ \\
\hline \multicolumn{6}{|l|}{ Need factors } \\
\hline \multirow[t]{3}{*}{ Subjective health level } & 0: Good & 4922 & 230 & 1.00 (reference) & 1.00 (reference) \\
\hline & 1: Mediocre & 9158 & 663 & $1.58(1.37,1.86)^{* * *}$ & $1.30(1.11,1.52)^{* *}$ \\
\hline & 2: Poor & 14111 & 2351 & $4.08(3.56,4.70)^{* * *}$ & $2.06(1.77,2.40)^{* * *}$ \\
\hline \multirow[t]{3}{*}{ No. of chronic diseases } & 0: None & 8405 & 853 & 1.00 (reference) & 1.00 (reference) \\
\hline & $1: 1-2$ & 11639 & 1258 & $1.07(0.97,1.17)^{* * *}$ & $0.83(0.75,0.91)^{* * *}$ \\
\hline & 2: 3 or more & 8147 & 1133 & $1.42(1.30,1.57)^{* * *}$ & $0.87(0.79,0.97)^{*}$ \\
\hline \multirow[t]{2}{*}{ Anxiety/depression } & O: No & 22426 & 1959 & 1.00 (reference) & 1.00 (reference) \\
\hline & 1: Yes & 55765 & 1285 & $3.00(2.77,3.24)^{* * *}$ & $1.87(1.71,2.03)^{* * *}$ \\
\hline \multirow[t]{2}{*}{ Pain/discomfort } & 0: No & 12440 & 688 & 1.00 (reference) & 1.00 (reference) \\
\hline & 1: Yes & 15751 & 2566 & $3.30(3.03,3.61)^{* * *}$ & $1.57(1.41,1.75)^{* * *}$ \\
\hline
\end{tabular}

Values are presented as odds ratio (95\% confidence interval). 
To determine whether the extent of unmet healthcare needs varied in the rural elderly depending on convenience of transportation, the subjects were divided into 3 groups: a group with no driver in the household (GR1), a group with a driver who was not the elderly (GR2), and the self-driving group (GR3), and the reasons for the level of unmet healthcare needs were described. SAS version 9.4 (SAS Institute Inc., Cary,
NC, USA) and R version 3.5.1 (https://cran.r-project.org/bin/ windows/base/old/3.5.1/) were used.

\section{Ethics Statement}

This study was conducted after obtaining approval with a review exemption from the Institutional Review Board of Seoul National University.

Table 3. Distribution of unmet healthcare needs stratified according to availability of individual transportation

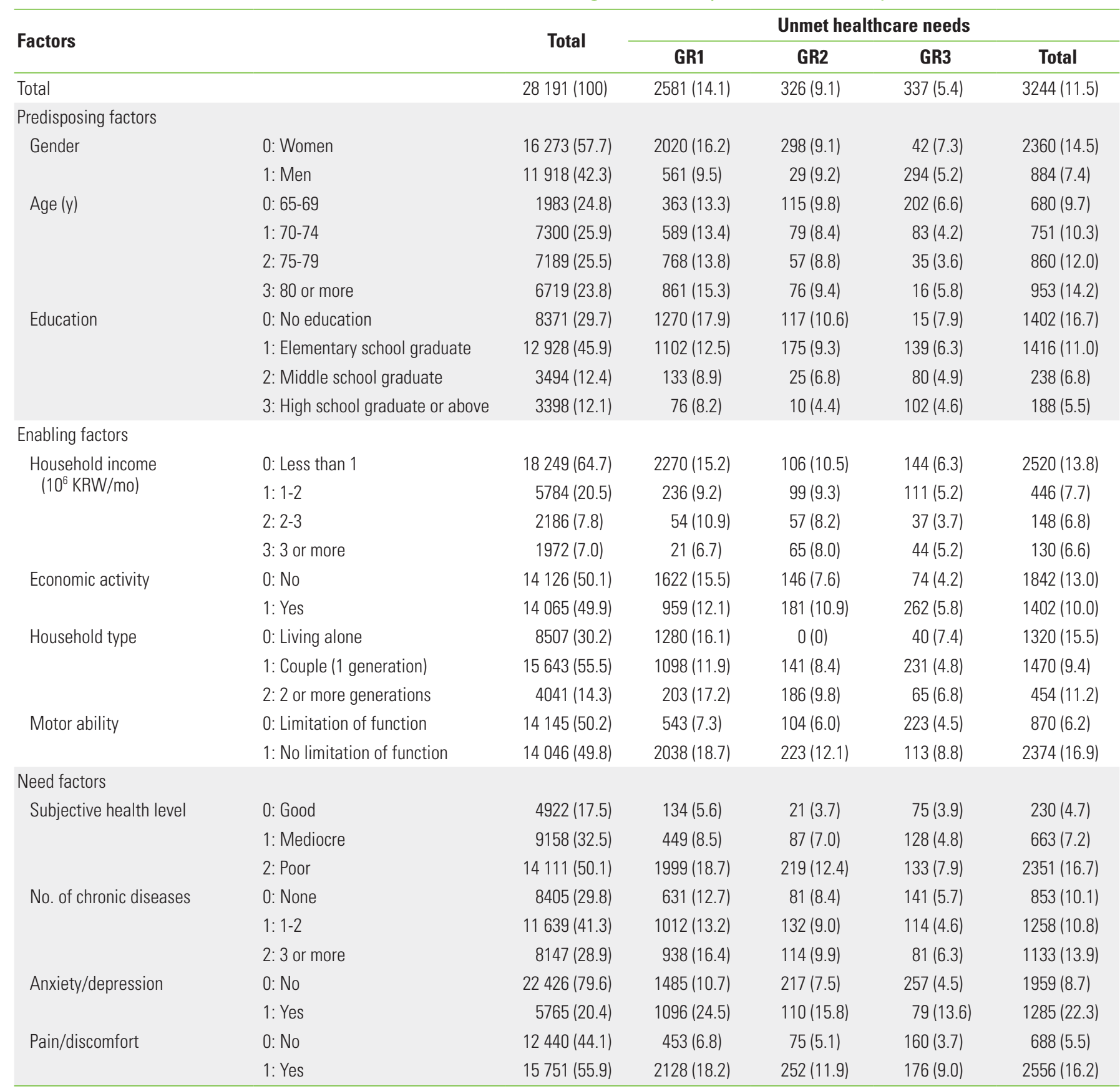

Values are presented as number (\%).

GR1, group with no driver in the household; GR2, group with a driver who was not the elderly; GR3, self-driving group; KRW, Korean won. 
Table 4. Distribution of reasons for unmet healthcare needs by group

\begin{tabular}{|c|c|c|c|c|}
\hline Characteristics & Total & GR 1 & GR2 & GR3 \\
\hline Total & $28191(100)$ & $18349(100)$ & $3580(100)$ & $6262(100)$ \\
\hline No unmet healthcare needs & $24947(88.5)$ & 15768 (85.9) & $3253(90.9)$ & $5926(94.6)$ \\
\hline Economic reason (financial accessibility) & $579(17.8)$ & $494(19.1)$ & $39(11.9)$ & $45(13.4)$ \\
\hline Transportation (physical accessibility) & $1157(35.7)$ & $1039(40.3)$ & $82(25.1)$ & $37(10.7)$ \\
\hline Other & $551(17.0)$ & $459(17.8)$ & $58(17.7)$ & $34(10.1)$ \\
\hline
\end{tabular}

Values are presented as number (\%).

GR1, group with no driver in the household; GR2, group with a driver who was not the elderly; GR3, self-driving group.

${ }^{1}$ Percentages of reasons are calculated using unmet health care needs of each group as 100 .

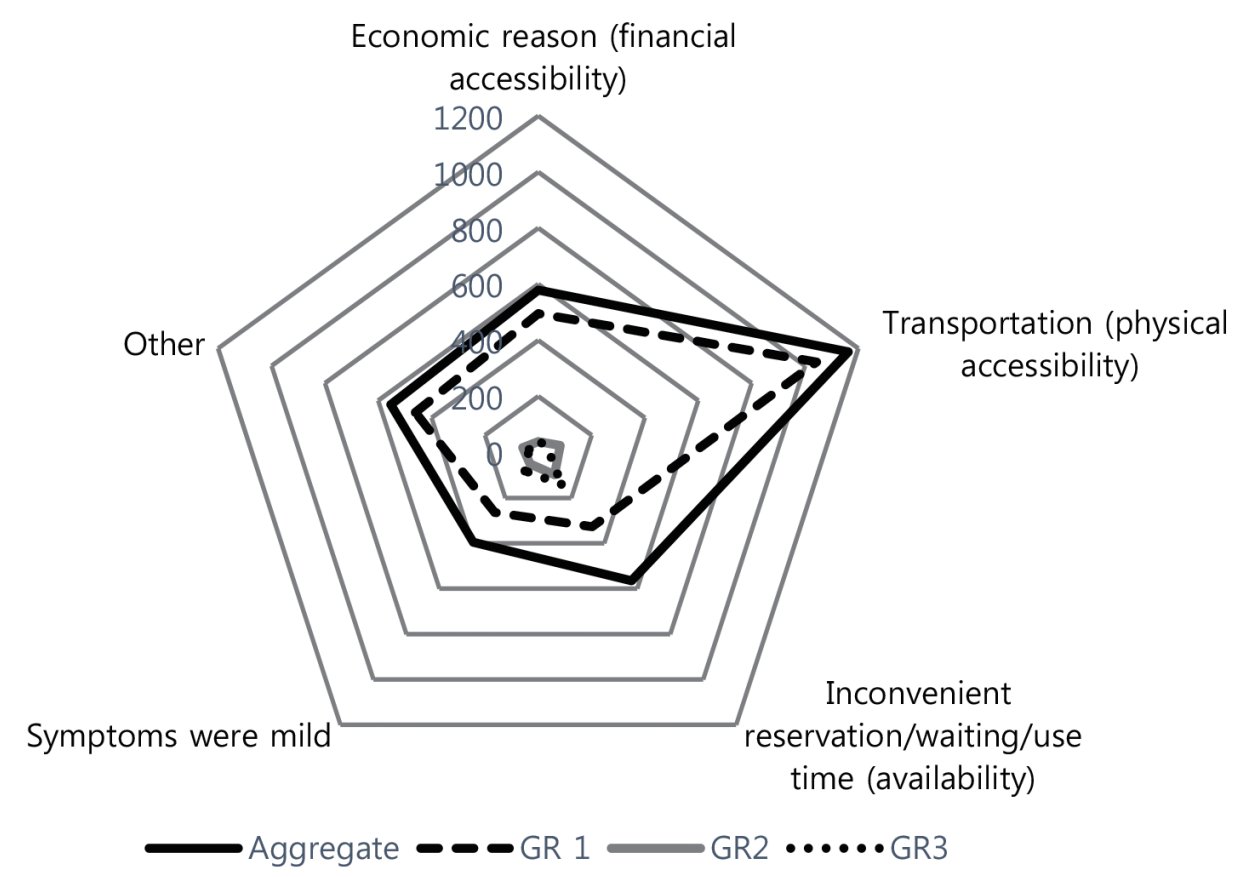

Figure 1. Reasons for unmet healthcare needs by group. GR1, group with no driver in the household; GR2, group with a driver who was not the elderly; GR3, self-driving group.

\section{RESULTS}

Table 1 presents general characteristics and descriptive statistical results associated with unmet healthcare needs among the rural elderly.

Table 2 and Supplemental Material 1 present the results of a logistic regression analysis. This shows the $\mathrm{OR}$ of each variable before and after adjustment of all factors.

The results of the stratification into 3 groups are presented in Table 3.

Table 4 and Figure 1 present the results of the distribution of the reasons for unmet healthcare needs by group.
The rates of unmet healthcare needs for GR1, GR2, and GR3 were $14.1 \%, 9.1 \%$, and $5.4 \%$, respectively. Regarding the reasons for these unmet needs, over $40.0 \%$ of the rural elderly in GR1 experienced unmet healthcare needs due to inconvenient transportation.

\section{DISCUSSION}

\section{Unmet Healthcare Needs of the Rural Elderly}

The elderly in the guns included were mostly women; combined, individuals with no formal education and an elementary school graduation accounted for $75.6 \%$, and individuals with 
less than 1 million Korean won (KRW) of monthly household income accounted for $64.7 \%$. These reveal vulnerabilities in multiple aspects. In comparison, in the National Survey of Senior Citizens [10], individuals with an education level of elementary school graduation or lower constituted $67.0 \%$, and their average annual income was 23 million KRW. With regard to driving, the proportion of self-driving individuals in our study was $22.2 \%$, while the proportions of those with a driver among family members and no driver among family members were $12.7 \%$ and $65.1 \%$, respectively. This proportion of selfdriving elderly individuals is slightly higher than the $16.1 \%$ obtained by the 2014 National Survey of Senior Citizens. However, given that the number of cars across the nation was over 20 million and the era of 2 cars per household started in 2016 [22], the rates of a driver's presence among family members and the availability of individual transportation were very low. The proportions of dysfunctional motor ability (49.8\%), and good subjective health level (17.5\%), the frequency of chronic diseases (70.2\%), and pain/discomfort (55.9\%) show high levels of healthcare necessity. In summary, while the barriers to visiting healthcare institutions by walking (due to disordered motor ability) and by using public transportation (due to its unavailability) were expected to be major, the availability of individual transportation was low, from which difficulty in finding transportation for the use of healthcare services is expected. Reflecting this, an overwhelming reason for unmet healthcare needs was inconvenient transportation. This is a striking discrepancy from the finding that economic reasons were the most frequent in a previous study associated with unmet healthcare needs $[5,23,24]$.

\section{Unmet Healthcare Needs of the Rural Elderly and Associated Factors}

Our study found that many factors were significantly associated with unmet healthcare needs in complex ways, and some particularly notable findings were confirmed. Regarding gender, women showed a higher level of unmet healthcare needs than men, which is consistent with the findings of previous studies [24-27]. Regarding age, while unmet healthcare needs increased with age before adjustment, the level of these needs decreased with age after adjustment. It can be concluded that, because unmet healthcare needs are more influenced by other factors that increase with age, such as chronic diseases, poor health level, restriction of motor ability, or pain, than by age itself, and that age itself lowers the level of unmet health needs.
In a study conducted by Moon and Kang [25], the young-old elderly displayed higher unmet healthcare needs than the oldold elderly. This was assumed to be true because the young-old elderly have relatively high expectations and more easily perceive unmet needs when those expectations cannot be satisfied. Regarding education level, unmet healthcare needs greatly decreased from the group with no formal education to the groups with an elementary, middle school, and high school education before adjustment, and it decreased until middle school graduation and slightly increased again until high school graduation and above after adjustment. The difference between before and after adjustment data can be explained through the concept that need factors decrease as health level increases along with education level, and higher education levels are associated with more resources that enable the use of healthcare, such as income and transportation. The post-adjustment phenomenon in which unmet healthcare needs decreased and then increased with education level can be explained by the fact that the highly-educated elderly have higher expectations for healthcare service beyond merely traveling to and visiting healthcare institutions. The study conducted by Kim and Lee [4] on the married migrant women showed that a higher education level corresponded with a higher level of unmet healthcare needs, and this example supports our explanation regarding levels of expectations.

Household income displayed results consistent with existing studies, as lower income was associated with a higher level of unmet health needs $[4,5,27]$. Regarding economic activity, before adjustment, unmet healthcare needs were lower when the elderly engaged in economic activity than when they did not. However, after adjustment, unmet healthcare needs were higher when the elderly engaged in economic activity. The pre-adjustment result is believed to be associated with the concept that economic activity is linked to high income and a lack of restriction of motor ability; after adjustment, however, unmet healthcare needs are believed to increase when the elderly engage in economic activity due to the corresponding lack of time. Existing studies have shown that economic activity is linked to unmet healthcare needs $[4,28]$. With regard to household type, before adjustment, the level of unmet healthcare needs was highest when the elderly individual lived alone and was lower in households where 2 or more generations cohabited, then decreased further in households where a couple cohabited. However, after adjustment and using living alone as a reference, the level of unmet healthcare needs was lower 
for cohabitation of a couple but was higher for cohabitation of 2 generations or more $(p<0.05)$. A previous study [22] showed that the level of unmet healthcare needs according to household type was highest in single-person households, lower in cases of cohabitation of a couple, and lowest in cohabitation with children. Elderly individuals who live alone experience difficulty when they visit hospitals alone, as they have a lack of social support [18]. They are also reluctant for their children with whom they do not live to take them to healthcare institutions because they fear burdening them [23]. As for why the level of unmet healthcare needs was higher in households where 2 or more generations cohabitated, the makeup of the household can play a role. In the past, 2-generation households usually consisted of adult parents and minor children or of elderly parents and adult children. However, considering the current population structure in rural areas, it can be assumed that households of 2 or more generations are mostly old-old support households consisting of old parents and old children.

Regarding the number of chronic diseases, which showed conflicting results in previous studies $[23,26,29]$, this implies that the elevated pre-adjustment level of unmet healthcare needs was caused by pain associated with chronic disease, worsening of subjective health level, or restrictions on motor ability, but the number of chronic diseases itself acted as a factor to promote more frequent hospital or clinic visits, lowering the level of unmet healthcare needs.

With regard to driving, the level of unmet healthcare needs was highest when there was no driver in the household both before and after adjustment. Before adjustment, unmet healthcare needs were lowest when the elderly individual could drive, but after adjustment, they were lowest when there was a driver in the household. The fact that unmet healthcare needs before adjustment was lowest when the elderly individual could drive may be the case because that group had relatively high income and high education and had no major restriction on motor ability. The reason why unmet healthcare needs for the self-driving group was relatively high after adjustment may be because the group had high expectations, similarly to what was proposed above regarding education level and the elderly who drive themselves may experience time restrictions due to economic activity or other obligations. Another reason is that when a family member drives, the level of unmet healthcare needs can decrease because relatively little inconvenience is associated with a guardian transporting a sick spouse or parents. A study in the USA [10] showed that healthcare use increased when a family member or a friend drove compared to self-driving.

\section{Differences Depending on the Convenience of Transportation}

In previous studies and when analyzed across the entire elderly sample, the level of unmet healthcare needs in women was high; however, when the groups were stratified, that in women was still high in GR1 and GR3, but slightly higher in men in GR2. Considering the gender and age distribution of driver's license holders in Korea, this is assumed to be because if there is a driver in a household, if the subject is woman, the driver is likely to be her husband, while if the subject is man, the driver is likely to be one of his children, rather than his wife. When household income was subdivided, for participants with a monthly income of less than 1 million KRW, the difference across groups was high, while for participants with an income of 3 million KRW or more, no association was found. The reason is believed to be that when household income is high, the household has other resources to secure transportation even though there is no driver. Regarding household type, a noteworthy result was obtained. In GR1, the level of unmet healthcare needs was $16.1 \%$ in elderly individuals living alone, and that figure was higher, at $17.2 \%$, in households of 2 or more generations. Many previous studies regarded the elderly living alone in rural areas as a vulnerable class, but the possibility should be considered that some households with cohabitants from 2 or more generations could also be vulnerable, such as old-old support households or grandparent-grandchildren households. With regard to motor ability, when no limitation of function was present, the level of unmet healthcare needs was relatively low (7.3\%) in GR1 but very high $(18.7 \%)$ when there was a limitation of motor function, constituting a large difference from GR2 and GR3. This means individuals who do experience a limitation of motor function, who are more influenced by the convenience of transportation because they have difficulty walking or using other inconvenient transportation methods. With regard to subjective health level, anxiety/depression, and pain/discomfort, GR1 displayed a higher level of unmet healthcare needs than GR2 and GR3, despite the presence of a need factor. This implies that individuals with high needs are highly influenced by the convenience of transportation.

Finally, the reasons for unmet healthcare needs varied across the 3 groups. In GR1, over $40.0 \%$ were attributed to a lack of 
transportation. GR1 is noteworthy because it had a much higher level of unmet healthcare needs that were caused by economic reasons, although GR1 would be expected to be quite similar to the other groups with regard to low income or socioeconomic level in general. Both GR2 and GR3 had low levels of unmet healthcare needs caused by a lack of transportation and relatively high levels of unmet healthcare needs caused by an inconvenient reservation, waiting, or use time. In a previous study focused on the reasons for unmet healthcare needs, the most prevalent reason was found to be economic $[27,30]$. The fact that the reason for unmet healthcare needs varies according to the characteristics of the group and the level of inconvenience of transportation is the most likely explanation for why the current study found different results for the level of unmet healthcare needs in the rural elderly than previous studies.

According to the 2016 Census of Agriculture, Forestry, and Fisheries, among agricultural and fishery villages, the percentage of villages with a health center less than a 10-minute drive away was just $44.6 \%$, and that with a hospital, clinic, or oriental clinic was just 31.5\% [31]. The percentages of areas with public transportation that operates 3 times or fewer per day and areas where it operates 6 times or fewer per day in all ri (village)-level administrative units as of 2015 were $11.9 \%$ and $40.0 \%$, respectively [32]. Many rural elderly who have no personal means of transportation walk for more than 1 hour or wait several hours for transportation to healthcare institutions.

In Korean studies conducted on unmet healthcare needs and accessibility to healthcare, the role of patients' transportation to healthcare institutions has received little attention. This is because the means of individual transportation have been regarded as private assets, unlike public transportation, so individual transportation has not been recognized as a subject impacting the healthcare field that could be addressed through policy measures. However, when working to increase accessibility to healthcare and to address various barriers, these barriers should be faced from a demander-centered viewpoint as opposed to the current provider-focused viewpoint, and efforts should be made to lower those barriers. In the UK, the National Health Service collects data on the types of transportation that can be used to travel to and from healthcare institutions [33] and the percentage of households that cannot use a vehicle in their healthcare institution accessibility index [34]. In Korea, the sharing model of transportation and demand-responsive transport have been introduced recently. However, the healthcare community should note that these practices are in early stages and are being executed as part of projects to improve life in rural areas, not as part of healthcare policy.

The limitations of this study are as follows. As a cross-sectional study the associations found in this analysis cannot be interpreted as influencing factor with a specific directionality. Next, this study only analyzed replies to survey questions. Thus, factors that were not investigated in the survey questions could not be assessed. Furthermore, because income level was measured as household income, if it were converted to per capita personal income, the values might differ from the measurements used in this study. In the Community Health Survey, an investigation should theoretically include all household members, but in reality, some household members can be omitted. Finally, when collecting and analyzing gunlevel data from 77 health centers, the convenience of transportation was used as a variable under the assumption that the status of public transportation and the movement paths to healthcare institutions were similar across all guns. Thus, it is a limitation that the differences between individual regions were not reflected in the results.

The inconvenience of transportation is a major barrier to the use of healthcare service by the rural elderly. Decision-makers must consider whether the barriers that must be overcome are the same regardless of region, rural area or city, age, and socioeconomic level [11], and must continuously work to find solutions for this problem. This study suggests that convenience of transportation is a factor involved in the accessibility of healthcare, and the results imply that transportation should be considered when developing measures to improve accessibility.

\section{SUPPLEMENTAL MATERIALS}

Supplemental material is available at https://doi.org/10. 3961/jpmph.19.172.

\section{CONFLICT OF INTEREST}

The authors have no conflicts of interest associated with the material presented in this paper.

\section{ACKNOWLEDGEMENTS}

This paper revises and complements $<A$ Study of Influencing Factors on Unmet Healthcare Needs of the Local Elderly in 
Korea >, the lead author's master's thesis at Graduate School of Public Health, Seoul National University.

\section{AUTHOR CONTRIBUTIONS}

Conceptualization:YC. Data curation: YC. Formal analysis:YC, KN. Methodology: YC, CYK. Funding acquisition: None. Writing - original draft: YC, KN. Writing - review \& editing: CYK, YC, KN.

\section{ORCID}

Youngeun Choi https://orcid.org/0000-0002-4065-7977

Kiryong Nam https://orcid.org/0000-0002-4253-6335

Chang-yup Kim https://orcid.org/0000-0002-4389-2454

\section{REFERENCES}

1. Chang DR, Moon OR. Equity of access to health services under national health insurance system in Korea. Health Policy Manag 1996;6:110-143 (Korean).

2. Lee YJ. A study on the inequality of health care resources distribution affected by regional characteristics. J Crit Soc Welf 2005;21(21):49-78 (Korean).

3. Ha RK. Factors affecting unmet health care needs of precarious workers [dissertation]. Seoul: Seoul National University; 2016 (Korean).

4. Kim SH, Lee CY. Analysis of factors affecting unmet healthcare needs of married immigrant women. J Korean Acad Nurs 2013; 43(6):770-780 (Korean).

5. Jeon BY, Kwon SM. Access barriers to health care among persons with physical disabilities: using three reasons for unmet need as indicators of access. Korean Soc Secur Assoc 2015; 31(3):145-171 (Korean).

6. Min DH, Cho JY, Kim JG, Seo SJ, Kim MK, Shim EH, et al. Medical experiences and unmet health care perception among elderly people with chronic disease. Health Policy Manag 2018; 28(1):35-47 (Korean).

7. Cho KS, Lee HJ. Accessibility of medical care in an elderly population. J Korean Acad Adult Nurs 2000;12(4):641-653 (Korean).

8. Ensor T, Cooper S. Overcoming barriers to health service access: influencing the demand side. Health Policy Plan 2004; 19(2):69-79.

9. Jacobs B, Ir P, Bigdeli M, Annear PL, Van Damme W. Addressing access barriers to health services: an analytical framework for selecting appropriate interventions in low-income Asian coun- tries. Health Policy Plan 2012;27(4):288-300.

10. Arcury TA, Preisser JS, Gesler WM, Powers JM. Access to transportation and health care utilization in a rural region. J Rural Health 2005;21(1):31-38.

11. Rosenthal TC, Fox C. Access to health care for the rural elderly. JAMA 2000;284(16):2034-2036.

12. Ricketts TC. Rural health in the United States. New York: Oxford University Press; 1999, p. 25-26.

13. Park JW, Moon JW. Welfare and transportation policy for the elderly in advanced foreign countries-focusing on the Japanese. Goyang: Korea Transport Institute; 2012 (Korean, author's translation).

14. Hwang J, Choi YJ. An analysis of the rural living condition of the elderly living alone. Korean Soc Community Living Sci 2013;11:119 (Korean).

15. Lee KJ, Seomoon JH. A study on the transportation welfare alternatives to enhance the right of mobility for the elderly in rural areas. Korean J Local Gov Adm Stud 2013;27(1):247-268 (Korean).

16. Yi YJ, Kim EJ. The effects of accessibility to medical facilities and public transportation on perceived health of urban and rural elderly: using generalized ordered logic model. J Korean Reg Dev Assoc 2015;27(1):65-88 (Korean).

17. Kim DJ. Status and implications of general and emergency medical use of rural residents. Issue Focus 2012;159:1-8 (Korean, author's translation).

18. Lee SH. Potential spatial access to healthcare for diabetic and hypertensive outpatients [dissertation]. Seoul: Seoul National University; 2013 (Korean).

19. An SA, Sim MY, Jeong BG, Kim JR, Kang YS, Park KS, et al. A study on elderly people in health inequality in vulnerable health areas centering around agriculture and fisheries areas. J Korean Gerontol Soc 2011;31(3):673-689 (Korean).

20. Whitehead M. Diffusion of ideas on social inequalities in health: a European perspective. Milbank Q 1998;76(3):469-492.

21. Aday LA, Andersen R. A framework for the study of access to medical care. Health Serv Res 1974;9(3):208-220.

22. Ministry of Land, Infrastructure and Transport. Infrastructure and transport; 2018 [citied 2018 Oct 10]. Available from: http:// www.molit.go.kr/USR/NEWS/m_71/dtl.jsp?id =95080239 (Korean).

23. Kim JG. Factors affecting the choice of medical care use by the poor. Korean J Soc Welf Stud 2008;37:5-33 (Korean).

24. Lee JE, Kim TH. Association of supplementary private health insurance with unmet health care needs. J Health Inform Stat 


\section{4;39(1):91-104 (Korean).}

25. Moon J, Kang M. The prevalence and predictors of unmet medical needs among the elderly living alone in Korea: an application of the behavioral model for vulnerable populations. Health Soc Welf Rev 2016;36(2):480-510 (Korean).

26. Song KS, Lee JH, Rhim KH. Factors associated with unmet needs for health care. Korean Public Health Res 2011;37(1):131-140 (Korean).

27. Kim SJ, Huh SI. Financial burden of health care expenditures and unmet needs by socioeconomic status. Korean J Health Econ Policy 2011;17(1):47-70 (Korean).

28. Jung YH. Limitation of activity and unmet healthcare needs in Korea Health Panel Study. Issue Focus 2012;120:1-8 (Korean, author's translation).

29. Kim KS, Lee HO. Household catastrophic health expenditure and unmet needs depending on the types of health care system. Soc Welf Policy 2012;39(4):255-279 (Korean).

30. Yu SY, Kim YS, Hong HS, Cheon MK, Mo JA. Determinants of untreated experiences among persons with physical disability. Health Policy Manag 2011;21(4):565-584 (Korean).
31. Statistics Korea. Results of the agriculture, forestry and fishery survey in 2016 [cited 2019 Nov 14]. Available from: http://kostat.go.kr/portal/korea/kor_nw/1/8/1/index.board?bmode $=r$ ead\&aSeq $=359996 \&$ pageNo $=6 \&$ row Num $=10 \& a m S e q=\& s T$ arget $=\& s T x t=$ (Korean).

32. Statistics Korea. Results of the 2015 census of agriculture, forestry and fisheries [cited 2019 Nov 14]. Available from: http:// kosis.kr/statHtml/statHtml.do?orgld=101\&tblld =DT_1AR15

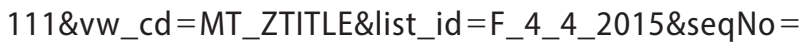
\&lang_mode $=$ ko\&language $=$ kor\&obj_var_id $=$ \&itm_id $=$ \&conn_path =MT_ZTITLE (Korean).

33. National Health Service. How do I organise transport to and from hospital? [cited 2018 Oct 10]. Available from: https:// www.nhs.uk/common-health-questions/nhs-services-andtreatments/how-do-i-organise-transport-to-and-from-hospital.

34. Jang DI, Kim CS, Lee SJ, Hong SP. Establishing transport accessiblity in metropolitan area. Goyang: Korea Transport Institute; 2015 (Korean). 